\title{
Mechanisms of perceptual timing: Beat-based or interval-based judgements?
}

\author{
Steven W. Keele, Roberto Nicoletti, Richard I. Ivry, and Robert A. Pokorny \\ Department of Psychology, University of Oregon, Eugene, Oregon 97403, USA
}

Summary. What is the nature of the human timing mechanism for perceptual judgements about short temporal intervals? One possibility is that initial periodic events, such as tones, establish internal beats which continue after the external events and serve as reference points for the perception of subsequent events. A second possibility is that the timer records the intervals produced by events. Later, the stored intervals can be reproduced or compared to other intervals. A study by Schulze (1978) provided evidence favoring beat-based timing. In contrast, our two experiments support an interval theory. The judgements of intervals between tones is not improved when the events are synchronized with internal beats established by the initial intervals. The conflict between the two sets of results may be resolved by the fact that an interval timer can recycle from one interval to the next, thus operating in a beatlike mode. However, a timer of this sort is just as accurate when comparing intervals that are off the beat.

A great deal of research has been concerned with the perception of time. Although "time" is a term in common, many of the problems addressed in different studies are quite different. Much of the work has been concerned with subjective time - that is, judgements of how much time has transpired. Typically those judgements concern seconds, minutes, and even hours. In contrast, little work has been concerned with relatively short intervals measured in terms of a few hundred ms. Such short intervals are characteristic of the timing of a rapid series of movements, as in playing a musical instrument or in speaking, and are also characteristic of the perceptual experiences emanating from rapid production of this sort, such as the music or the speech sounds.

The present study is concerned with possible mechanisms that underlie the perception and production of such short intervals as may be relevant to skill. In particular, the study compares two general mechanisms whereby humans distinguish slight irregularities in the timing of otherwise periodic events. The events occur at short intervals, in this case one about every $300 \mathrm{~ms}$. The two mechanisms were described by Schulze (1978). One mechanism, a beat-based timer, essentially involves the synchrony of events. The second type of mechanism, an interval timer, involves

Offprint requests to: $\mathrm{S}$. W. Keele comparing the intervals between events. The general idea of a beat-based timer is that an initial series of periodic events establishes an internal beat that persists after the initial events. Whether or not subsequent events are perceived as occurring at the proper time will depend on the degree to which they are synchronized with the internal beat. In contrast, an interval timer registers the duration of an interval between events, and that temporal memory is then compared to other intervals in order to judge whether or not they are the same.

Schulze (1978) devised a test between the theories using a paradigm which we repeat here. On each trial he presented a series of either seven or nine tones forming a succession of six or eight intervals (we shall describe only the seven-tone case, since the results were the same in both conditions). The subjects' task was to decide whether the intervals produced by the tones were all equal or whether they differed. The first two intervals were always $300 \mathrm{~ms}$, and in the control situation that interval continued for the entire sequence. Let us represent that sequence as:

\section{$\mathrm{t} t \mathrm{t} t \mathrm{tt}$}

where $t$ is an interval equal to $300 \mathrm{~ms}$. In the first of the unequal cases (Condition 1), the first two intervals were equal, but the following ones were all incremented by either 10 or $15 \mathrm{~ms}$. That condition may be represented as:

$$
\text { (1) } t \mathrm{t} t+\mathrm{t}+\mathrm{t}+\mathrm{t}+
$$

In Condition 2, only the interval that followed the two standards was incremented. The remaining intervals returned to the standard value:

(2) $t \mathrm{tt}+\mathrm{ttt}$.

Finally, in Condition 3, an increment in the third interval was followed by an equal decrement in the fourth interval. The final two intervals were equal to the standard:

(3) $t \mathrm{t} t+\mathrm{t}-\mathrm{t} \mathrm{t}$

Again, the subjects' task was to determine whether or not all intervals were equal. The issue concerns which of the three unequal conditions is easiest to discriminate from the all-equal condition. The prediction varies with the model of the timekeeper.

Schulze proposed two versions of an interval timer. In one version, which we call the adjacency model, adjacent intervals are compared. According to this model, Condition 3 should be the easiest, because it contains three pairs 
of adjacent intervals that are different in duration. Condition 2 provides only two differences between adjacent intervals and Condition 1 provides only one difference. Furthermore, the difference between the third and fourth intervals in Condition 3 is twice as large as any difference in the other conditions.

Another interval theory, which we call the memory model, makes a different prediction. By this theory, a memory trace representing the perceived average of the first two standard intervals is stored and subsequent intervals are compared to the stored standard. Condition 1 should be the easiest, then Condition 3, and Condition 2 should be the most difficult. The reasoning is that the memory trace differs from four comparison intervals in Condition 1, from two comparison intervals in Condition 3, and from only one interval in Condition 2.

The final theory that Schulze considered was a beat theory. The idea is that the first three tones that bound the first two intervals establish an internal beat that continues afterward. Because subjects know that the first tones establish the basic beat, it is assumed that later tones, regardless of when they occur, do not alter the beat. The time of occurrence of the subsequent tones can be compared with the time of occurrence of the internal beat. According to this theory, Condition 1 should be easiest, followed by Condition 2 and then Condition 3 . The reason is that for Condition 1 the internal beat will be out of synchrony with the time of the first comparison tone by $10 \mathrm{~ms}$ (for the case in which the increment is $10 \mathrm{~ms}$ ). The second comparison tone will be out of synchrony by $20 \mathrm{~ms}$, the third tone by $30 \mathrm{~ms}$, and the final tone by $40 \mathrm{~ms}$. In other words, once the internal beat is established, its asynchrony with the externally occurring tones will grow as long as the external intervals all continue to be larger by $10 \mathrm{~ms}$. For Condition 2 , the first incremented interval will produce an asynchrony of $10 \mathrm{~ms}$, but since the remaining intervals drop back to normal, the asynchrony initiated with the single increment will maintain itself at $10 \mathrm{~ms}$ for all remaining tones, producing four pulses in which the external tone is $10 \mathrm{~ms}$ out of synchrony with the internal beat. Because the asynchrony doesn't grow, this second condition should be more difficult to discriminate from equality than the first one. Condition 3 should be the most difficult, because while the first comparison tone produces an asynchrony with the internal beat, the second comparison tone restores synchrony.

The results of Schulze's experiment favored the beat theory. Condition 1 was the easiest and there was a tendency for Condition 2 to be easier than Condition 3 . However, in only one of four comparisons was the contrast between Conditions 2 and 3 reliable. Thus, in a statistical sense, the data by and large were also consistent with the memory theory in which a standard is compared to other intervals. Our own results show a significant difference between Conditions 2 and 3 and one that favors a memory theory (see below). A possible solution to the discrepant results will be considered in the discussion.

Schulze's experiment clarifies the meaning of a beatbased timer. It refers to a timekeeper similar to a metronome in which initial events set up an internal rhythm that serves as a benchmark against which to judge other events. In contrast, a memory-interval timer can measure intervals that start at arbitrary times, rather than being synchronized with the hypothetical internal rhythm. This mecha- nism is analogous to a stopwatch. Put another way, a beatbased timer involves comparisons of the time of events. An interval timer involves comparisons of the durations of intervals.

Our motivation for reexamining the issue of timing mechanism stemmed from work on patients that seemed more consistent with an interval timer. This work was based on a model of timing proposed by Wing and Kristofferson $(1973$; Wing, 1980) that posits a separation between an internal clock that generates intervals and the motor processes that implement movement. Wing, Keele, and Margolin (1984) described a Parkinson patient (with presumed basal-ganglia damage) who had difficulties with the clock component. In subsequent studies we (Ivry \& Keele, 1989; Ivry, Keele, \& Diener, 1988) also implicated the cerebellum and the cortex in the clock. Since a clock disturbance occurred in disparate neural systems, we (Ivry \& Keele, 1989) speculated that the timer is best conceived as a loop in which the request for motor activity at a particular time originates in the cortex, is passed to the cerebellum where a timing process meters out the appropriate temporal delay, and is passed back to the cortex for implementation (other evidence developed in Ivry and Keele, 1989 , and Ivry, Keele, and Diener, 1988, provides the reasons for assigning the central timing component to the cerebellum). It is only after the cortex receives the signal back from the cerebellum that a motor response may be initiated. Another pass through the clock loop can be started at that time to meter out either the same or a different interval. That is, the timer consists of an adjustable delay line rather than an oscillator. A similar delay-line conception was first proposed by Braitenberg (1967) for the cerebellum.

Presumably, the delay line that produces a particular interval can be drawn upon from any arbitrary time. Thus, while not incontrovertible, our patient data suggested a delay line or interval timer rather than an oscillatory or beat timer and prompted us to replicate Schulze's study.

\section{Experiment 1}

The procedure was much the same as that of the Schulze experiment except that we used slightly longer increments and decrements to the base-time interval. Whereas he used changes of 10 and $15 \mathrm{~ms}$, we used changes of $20 \mathrm{~ms}$, since in pilot work we found that unpracticed subjects had excessive difficulty with the smaller differences.

\section{Method}

Subjects. Seventeen young adults, mostly university students, were paid $\$ 4.00$ for participating in a one-session experiment. There were no special requirements for participation other than professed normal hearing.

Procedure. Each subject listened to a total of 270 series of seven tones. For each series the subject judged whether the intervals between the tones were all equal or whether some were different than others.

The tones were generated on a computer and played through a small speaker sitting about $50 \mathrm{~cm}$ in front of the subject. They were $10 \mathrm{~ms}$ in duration, 1,000 hertz, and approximately $50 \mathrm{db}$ in intensity. Because of the short duration, the tone had a click quality. 
The tone series were of four types. In one case all the intertone intervals were equal at $300 \mathrm{~ms}$. In the other three cases, the first two intervals were $300 \mathrm{~ms}$, but some of the other intervals differed. Subjects were told that the first two intervals were constant at a standard length and that the intervals that followed could be the same or different. In Condition 1 , represented as $\mathbf{t} t \mathbf{t}+\mathbf{t}+\mathbf{t}+\mathbf{t}+$, the last four intervals were $320 \mathrm{~ms}$ in duration, an increment of $20 \mathrm{~ms}$ over the standard. In Condition $2, \mathrm{t} t \mathrm{t}+\mathrm{t} \mathrm{t} t$, only the third interval was incremented, again by $20 \mathrm{~ms}$. In Condition 3 , $\mathrm{t} \mathrm{t} \mathrm{t}+\mathrm{t}-\mathrm{t} \mathrm{t}$, the third interval was incremented $20 \mathrm{~ms}$ over the standard, and the fourth interval was decremented. In a block of 90 trials, one half of the series involved all equal intervals and the other half involved one of the three conditions of inequality. The first 30 of the 90 trials were for practive and the last 60 were analyzed. The order of the three blocks of trials, each block having a different condition of inequality, was randomized over subjects.

After a tone series, a subject pressed either the $E$ (for equal) or $U$ (for unequal) key on the computer keyboard. The subject then pressed the space bar on the keyboard to initiate the next trial. The first of the tones occurred $1 \mathrm{~s}$ after the space-bar press.

\section{Results and discussion}

Averaged over all subjects, the percentage correct on trial blocks involving the $\mathrm{t} t \mathrm{t}+\mathrm{t}+\mathrm{t}+\mathrm{t}+$ condition was 87 ( $89 \%$ correct when all intervals were equal and $84 \%$ correct on the unequal trials). The percentage correct for blocks involving the $\mathrm{t} t \mathrm{t}+\mathrm{t} \mathrm{t} \mathrm{t}$ condition was $77(81 \%$ correct on the equal trials and $74 \%$ correct on the unequal trials). For the $\mathrm{t} t \mathrm{t}+\mathrm{t}-\mathrm{t} \mathrm{t}$ condition, the percentage correct was 82 ( $83 \%$ correct on the equal trials and $81 \%$ correct on the unequal trials). Since percentages were used, an arcsin transformation was performed before the data were entered into an analysis of variance. The ANOVA showed a significant difference among conditions, $F(2,32)=6.42$, $p<.005$. Newman-Keuls tests showed the difference between all conditions to be significant at or beyond the .05 level of confidence.

Schulze (1978) presented his data in terms of d', based on signal-detection theory. Some of our subjects were $100 \%$ correct for some trial types so that d' could not be calculated. Nonetheless, it is possible to tell whether d' is greater in some conditions than others, despite $100 \%$ performance. For example, if the percentage correct in one condition was 100 on the same trials and 90 on the different trials (i.e., $10 \%$ false alarms), d', though not calculable, would be greater than a case with corresponding $95 \%$ hits and $10 \%$ false alarms. Using this approach, we counted the number of times in which d' in one condition exceeded that in another, and applied the sign test. Condition $1(t \mathrm{t} \mathrm{t}+\mathrm{t}+\mathrm{t}+\mathrm{t}+)$ exceeded Condition $2(\mathrm{t} t \mathrm{t}+\mathrm{t} t \mathrm{t})$ for 15 of 16 subjects and there was one tie $(p<.05)$. Condition 1 exceeded Condition $3(t) t+t-t$ t) for 12 of 16 subjects and there was one tie $(p<.05$ by a one-tailed sign test). While Condition 3 exceeded Condition 2 for 10 of 17 subjects, the sign test was not significant.

The pattern of results is similar to, but not completely the same as, that of Schulze. He too found judgements the easiest when all intervals following the standards were incremented. However, he found that the second condition, the one involving a single incremented interval, was easier than the third involving one incremented and one decremented interval. That latter difference was statistically reliable in only one of four comparisons in his study. The opposite difference in the present study was reliable by a parametric test on percentage correct, though it failed on a less powerful nonparametric test on d'.

The results from our study support the memory-interval theory rather than the beat theory. These two theories are distinguished by the second and third conditions. By a beat theory, the third condition should be the more difficult because, although the incremented interval puts the internal beat temporarily out of synchrony with the series of tones, the next interval restores synchrony. Thus, the sequence should be relatively difficult to distinguish from equality, since only one event is displaced from the internal beat. In the second condition the incremented interval is not compensated by the following interval, and so the remaining tones stay out of synchrony with the hypothesized internal beat. In contrast, by the memory-interval theory, Condition 3 should be easier than Condition 2. According to that theory, a record of the initial standard intervals is compared to the durations of succeeding intervals. Since Condition 3 has two intervals discrepant from the standard, it should be more easily discriminated from equality than Condition 2.

With regard to another interval theory, the adjacency theory, the current results are consistent with those of Schulze in rejecting it. In that theory adjacent intervals are compared. Such a theory would predict Condition 3 to be the easiest, which is not the case.

Because the current results are not consistent in complete detail with those of Schulze, it is important to replicate the study. Replication is especially necessary since the difference between the critical Conditions 2 and 3 was not sufficiently strong. Experiment 2 provides a replication, but with a useful variation.

\section{Experiment 2}

In this experiment subjects first listened to four successive tones separated by $300 \mathrm{~ms}$. The resulting three intervals constituted a standard. After a pause of either 540 or $660 \mathrm{~ms}$, they heard four more tones. The task was to judge whether or not all the intervals in the second part of a series were equal in length to those in the first part. The base condition consisted of three intervals after the pause, all of which were equal to the standard. In Condition 1 , the following three intervals were all longer than the standard by $25 \mathrm{~ms}$ (the increment was increased from that of Experiment 1 because of more difficult discrimination when a pause is inserted in the tone series). In Condition 2 the first interval after the pause was incremented by $25 \mathrm{~ms}$, and the remaining two intervals were equal to the standard of $300 \mathrm{~ms}$. In Condition 3 the first interval following the pause was incremented by $25 \mathrm{~ms}$, the second interval was decremented by $25 \mathrm{~ms}$, and the remaining interval was $300 \mathrm{~ms}$. Except for the pause and the size of the increments, the conditions were as in Experiment 1.

The purpose of the pause was to put an internal beat out of synchrony with the events to be judged. Assuming that the initial four tones establish an internal beat that occurs every $300 \mathrm{~ms}$, then the first tone after a pause of $540 \mathrm{~ms}$ would occur $60 \mathrm{~ms}$ before the beat. In Condition 1 , in which all of the intervals after the pause are incremented, the second tone after the pause would occur $35 \mathrm{~ms}$ be- 
fore, the next tone $10 \mathrm{~ms}$ before, and the final tone $15 \mathrm{~ms}$ after the beat. Thus, the initial discrepancy between the internal beat and the tone would tend to decrease as the series of comparison tones progressed. In contrast, following a 660 -ms pause, the first tone of the test series would be $60 \mathrm{~ms}$ out of synchrony with the supposed internal beat, and the asynchrony of succeeding tones would grow to 85 , 110 , and $135 \mathrm{~ms}$. Thus the beat theory would predict that the judgements of Condition 1 would be much easier after a pause of $660 \mathrm{~ms}$, where initally large asynchronies would grow, than after a pause of $540 \mathrm{~ms}$, where initially large asynchronies would diminish.

It could be argued, however, that an initial series of events selects an oscillator of sorts that stops during the pause. The initial tone after the pause restarts the oscillator, and its beats are then compared with the successive tones. If this is the case, then no difference should be expected between the conditions of long or short pause. The experiment would then simply replicate Experiment 1.

\section{Method}

Subjects. Ten young adults from the subject pool of the Cognitive Laboratory were paid \$ 8.00 each for participation in two 1-hour sessions. Again, most were students and no special requirements were necessary for participation. None of the subjects had participated in Experiment 1.

Procedure. The apparatus and materials were the same as in Experiment 1, with two exceptions. First, eight rather than seven tones were presented in each series. Second, a pause of either 540 or $660 \mathrm{~ms}$ was inserted between the fourth and fifth tones. Each subject participated in each of the six combinations of three conditions by two pause durations. In a block of 90 trials, half of the tone series involved all-equal intervals (with the exception of the pause) and the other half involved one of the three increment types: all intervals subsequent to the pause were incremented, one was incremented, or one was incremented and the next was decremented. The base interval established by the first three intervals before the pause was $300 \mathrm{~ms}$, and any increment or decrement to the base interval was always $25 \mathrm{~ms}$. The two pause intervals were run in separate sessions and were counterbalanced across subjects. Within a session, there was one block of 90 trials for each of the increment conditions. The first 30 trials of each block of 90 were considered practice, and all the data reported are based on the last 60 trials of the block. The order of conditions within a session was randomized for each subject.

\section{Results and discussion}

For the condition with a $540-\mathrm{ms}$ pause between the standard intervals and the comparison intervals, the percentage correct averaged over subjects and over both the same and different trials was 83 for Condition 1 in which all comparison intervals were incremented, 76 for Condition 2 in which only the first comparison interval was incremented, and 81 for Condition 3 in which the first comparison interval was incremented and the second decremented. For the 660-ms pause the respective scores were 82 , 69 , and $76 \%$. An analysis of variance based on an arcsin transformation of the percentages showed a significant effect of condition of incrementing, $F(2,18)=8.21$,
Table 1. Percentage correct and d' for different temporal conditions and for trials on which all intervals were equal or trials on which some intervals differed from the standard

\begin{tabular}{|c|c|c|c|}
\hline \multirow[t]{2}{*}{ Condition } & 1 & 2 & 3 \\
\hline & $(\mathrm{t} t \mathrm{t}+\mathrm{t}+\mathrm{t}+\mathrm{t}+)$ & $(t \mathrm{t} t+\mathrm{t} t \mathrm{t})$ & $(t t t+t-t t)$ \\
\hline$\%$ Correct & & & \\
\hline $\begin{array}{l}\text { All-equal } \\
\text { intervals }\end{array}$ & 84 & 79 & 82 \\
\hline $\begin{array}{l}\text { Unequal } \\
\text { intervals }\end{array}$ & 81 & 65 & 74 \\
\hline Mean & 82 & 72 & 78 \\
\hline Mean d'* & 2.23 & 1.34 & 1.71 \\
\hline
\end{tabular}

* d' is calculated separately for each subject and then averaged to obtain the mean d'

$p<.005$, but neither the effect of pause duration, $F(1,9)=0.62$, nor the pause by condition interaction, $F(2,18)=0.25$, was significant. A transformation of percentage data, such as by the arcsin transformation, could change the form of an interaction, but it is clear that the original untransformed data show more or less the same patterns across the two pause types. Nevertheless, the analysis was repeated on the raw percentages without the application of the arcsin transformation. The results were the same, with a significant main effect of condition of incrementing $(p<.005)$ and no other significant effect. Given no significant differences in the two pause types, further comparisons can be based on the two pause types averaged together.

Table 1 summarizes data averaged over the two pause types. Newman-Keuls tests on the mean percentage correct with arcsin transformation showed that all three conditions of incrementing were significantly different from each other at, or beyond, the .05 level of confidence. In this experiment no subject was $100 \%$ correct on any trial type, so the data could be converted to the signal detection measure of d'. Such a d' conversion was made for all subjects and then an analysis of variance was applied. The overall analysis showed reliable differences among the conditions $(p<.001)$. A post-hoc analysis with NewmanKeuls tests showed a reliable difference between Conditions 1 and 3 and 1 and 2, but the critical difference between Conditions 2 and 3 was not significant at the .05 level $(p<.10)$.

Part of the slight discrepancy between the analyses based on percentages and on d' may be due to the fact that some subjects had performance at, or above, $95 \%$ correct for either the all-equal pattern, the unequal pattern, or both. This was especially true in incremental Condition 1 where in general performance was highest. When percentages approach $100 \%$, very small percentage differences can make sizable differences in d'. Thus, relatively speaking, the d's for extreme percentages tend to be unreliable, which in turn increases the error-variance term in the analysis of variance. Since the Newman-Keuls post-hoc tests made use of the error variance from the overall ANOVA, those tests too were rather conservative. This problem is reduced when a post-hoc analysis of variance is applied only to the d's of Conditions 2 and 3 . In that case the difference is significant, $F(1,9)=7.07, p<.025$. 
The pattern of results thus replicated that of Experiment 1 , but again the critical comparison of Conditions 2 and 3 is not sufficiently strong from a statistical viewpoint. Both experiments show significant differences when percentages correct are compared, but the results are more marginal when d's are compared. Thus it is of interest that in another experiment we have conducted (Nicoletti \& Keele, 1988), using a method identical to that of Experiment 1 , very similar results were again obtained. The overall percentage correct in Conditions 1, 2, and 3 were 84,69 , and 77 , respectively. Again an analysis of variance on arcsin transformations was significant, and a NewmanKeuls test on the difference between Conditions 2 and 3, the two conditions of prime interest, was also significant $(p<.05)$. That study, however, did not report a d' analysis, because some subjects had $100 \%$ correct on some trial types. Given significant differences between Conditions 2 and 3 in all three experiments when percentages are considered, and similar trends with marginal significance when d's are considered in the two experiments here, it is clear that in general Condition 3 produces a better performance than Condition 2.

Two aspects of the present results are inconsistent with a beat theory. First, this type of timer would predict greater difficulty in discriminating Condition 1 from the allequal condition for a pause of $540 \mathrm{~ms}$ than for a pause of $660 \mathrm{~ms}$. The reason is that for the $540-\mathrm{ms}$ pause the first tone of the comparison series would occur before the hypothetical internal beat, and the asynchrony would diminish with each successive tone. With the $660-\mathrm{ms}$ pause the initial asynchrony would increase rather than diminish. However, the 540-ms pause of Condition 1 was not more difficult than with the $660-\mathrm{ms}$ pause, invalidating the beat model. Second, even if it is argued that the first event after a pause re-initialized the beat, the easier discriminability of Condition 3 in comparison to that of Condition 2 also speaks against the beat theory.

The current results are more consistent with the memory-interval theory that Schulze (1978) considered, but rejected. According to that theory initial intervals establish a record which can then be compared at arbitrary times to other intervals. The greater the number of comparison intervals that differ from a standard, the easier it is to discriminate the change. Whether or not the events that mark an interval fall in synchrony with the timing established by preceding events is of no consequence in this theory. The temporal memory can be employed at arbitrary times whenever a comparison is required.

\section{General discussion}

Both experiments in this study are consistent in suggesting that judgements of temporal equivalence are based not on synchrony of events with internal beats, but on a memory for interval durations. In turn, the results are consistent with our analyses of patients (Ivry \& Keele, 1989; Ivry, Keele, \& Diener, 1988) that suggest a timing mechanism distributed over several neural systems rather than an oscillator that emits signals from a small local area of the nervous system. In addition, the experiments are consistent with those of Schulze (1978) in rejecting a theory in which adjacent intervals are compared.

The rejection of the adjacency-interval theory is of interest in light of an experiment by Nicoletti and Keele
(1988). Subjects listened to a series of seven tones, as in Experiment 1 here. In an intensity condition they judged whether or not the tones were all of equal intensity. In a frequency condition, they judged whether or not the tones were of equal frequency. For those two types of judgement the presence of an adjacent contrast in which one tone was incremented (either on intensity or frequency) and the next was decremented made the differences especially discriminable. For the frequency condition the variations of adjacent intervals produce a kind of melody, and such seems to be the case for intensity as well. Temporal variation, in contrast, does not seem to produce a kind of "melody" in which adjacent intervals have special status, since having especially large differences between adjacent intervals did not enhance discriminability.

The question remains, why our results differ from those of Schulze (1978). We have pointed out that his results are ambiguous from a statistical standpoint, but that is not an entirely satisfactory explanation. The distinction between beat theory and the memory-interval theory rides on the difference in outcome of Conditions 2 and 3 . Schulze had four such comparisons and, though only one of them significantly favored the beat theory, all four comparisons were in the direction of favoring beat theory. Thus it is quite likely that his results also constitute a reliable outcome. How then might the conflicting results in two otherwise comparable studies be reconciled?

One possible reconciliation is suggested by experiments briefly mentioned in Keele (1987, pp. 482-483). In those experiments subjects heard a train of tones. After the last tone a signal was presented telling subjects to tap twice to reproduce the interval between the tones. Some subjects, but not all, started their tapping as though they were synchronizing with an internal beat. Thus, when the interval between tones was lengthened, those subjects delayed the onset of the first tap by about the same amount as the interval lengthening. When the signal to tap occurred early, they delayed their first tap slightly, rather than responding immediately. It is reasonable to assume that these subjects were waiting for beat synchronization.

Despite the fact that some subjects appeared to synchronize interval production with a beat, other subjects showed no such tendency. Moreover, even those who showed synchronization could easily abandon it. For example, when the instructions required subjects to begin the reproduction taps as soon as possible after the reproduction signal, the tendency to synchronize disappeared. Even so, there was no loss of accuracy in reproduction of the interval. Such results suggest that any tendency to base temporal decisions on internal beats is purely strategic. In fact, it is possible that subjects on occasion externalize a beat strategy by setting up an explicit movement, such as a tap of the foot or a nod of the head, in time with a periodic event. They may continue the external beat after the initial events and then use that movement as a base for judgements. If these arguments are valid, Schulze's results may have been due to his subjects' adoption of a nonobligatory beat strategy. We do not know of any differences in Schulze's study as compared with this one that may have inclined his subjects toward a beat-based strategy. Given his relatively small number of subjects, however, he may by chance have selected some who preferred that strategy.

If either timing mechanism is possible, interval or beat, then which is the most basic? One possible solution is to 
realize that an interval timer can operate in a beat mode. If a timed interval is recycled from end to beginning, then in essence it can mimic a beat mechanism. If our neurological notions about timing (Ivry \& Keele, 1989) are correct, and the clock system involves a neural loop from cortex to cerebellum and back to cortex, then subjects might produce internal beats by continually cycling through the loop. However, despite a capability of operating in a beat mode, the present experiments demonstrte that temporal judgements are not restricted to beat synchrony. Our hypothesized loop times can be used as an interval measure to compare with other intervals, whether they are periodic or not and whether or not they are isolated in time from other intervals.

In connection with this proposal, it is worth noting the results of Vorberg and Hambuch $(1978,1984)$. They concluded that, at least for simple serial intervals of equal length, successive intervals are generated by concatenation rather than being synchronized with a periodic internal beat. This is similar to the present suggestion that subjects can generate an apparent beat by concatenating successive intervals, but such intervals are not further synchronized with some other beat source.

Acknowledgements. Roberto Nicoletti, now at the University of Padua in Padua, Italy, was supported by a fellowship from the Consiglio Nazionale delle Ricerche of Italy. Richard Ivry is now at the University of California, Santa Barbara. Robert Pokorny is now at the AFHRL, Brooks Air Force Base in San Antonio, Texas. The research was supported by the Office of Naval Research contracts N00014-83-K-0601 and N0014-87-K-0279 to Michael Posner and Steven Keele.

\section{References}

Braitenberg, V. (1967). Is the cerebellar cortex a biological clock in the millisecond range? Progress in Brain Research, 25, $334-346$.
Ivry, R. I., \& Keele, S. W. (1989). Timing functions of the cerebellum. Cognitive Neuroscience, $1,134-150$.

Ivry, R. I., Keele, S. W., \& Diener, H. (1988). Differential contributions of the lateral and medial cerebellum to timing and to movement execution. Experimental Brain Research, 73, $167-180$

Keele, S. W. (1987). Sequencing and timing in skilled perception and action: An overview. In A. Allport, D. Mackay, W. Prinz, \& E. Scheerer (Eds.), Language Perception and Production. New York: Academic Press, pp. 461-487.

Nicoletti, R., \& Keele, S. W. (1988). Confronto di differenti modelli sul riconoscimento di sequenze di intervalli temporali. Giornale Italiano di Psicologia, 15, 89-100. (Available in English translation as: Comparison of different models of perception of sequences of temporal intervals. Technical Report, 1989, Cognitive Science Institute, University of Oregon, Eugene, Oregon, USA.)

Schulze, H.- H. (1978). The dectectability of local and global displacements in regular rhythmic patterns. Psychological Research, 40, 173-181.

Vorberg, D., \& Hambuch, R. (1978). On the temporal control of rhythmic performance. In J. Requin (Ed.), Attention and performance VII. Hillsdale, New Jersey, USA: Erlbaum, pp. 535-555.

Vorberg, D., \& Hambuch, R. (1984). Timing of two-handed rhythmic performance. In J. Gibbon and L. Allan (Eds.), Timing and time perception. Annals of the New York Academy of Sciences, 423, 390-426.

Wing, A. M. (1980). The long and short of timing in response sequences. In G. E. Stelmach \& J. Requin (Eds.), Tutorials in motor behavior. Amsterdam: North-Holland, pp. 469-486.

Wing, A. M., Keele, S. W., \& Margolin, D. I. (1984). Motor disorder and the timing of repetitive movements. In J. Gibbon \& L. Allan (Eds.), Timing and time perception. Annals of the New York Academy of Sciences, 423, 183-192.

Wing, A. M., \& Kristofferson, A. B. (1973). Response delays and the timing of discrete motor responses. Perception \& Psychophysics, 14, 5-12.

Received August 5, 1988/December 8, 1988. 